\title{
Analysis of a No Equilibrium Linear Resistive-Capacitive-Inductance Shunted Junction Model, Dynamics, Synchronization, and Application to Digital Cryptography in Its Fractional-Order Form
}

\author{
Sifeu Takougang Kingni, ${ }^{1}$ Gaetan Fautso Kuiate, ${ }^{2}$ Romanic Kengne, ${ }^{3,4}$ \\ Robert Tchitnga, ${ }^{3,4}$ and Paul Woafo ${ }^{5}$ \\ ${ }^{1}$ Department of Mechanical and Electrical Engineering, Institute of Mines and Petroleum Industries, University of Maroua, \\ P.O. Box 46, Maroua, Cameroon \\ ${ }^{2}$ Department of Physics, Higher Teacher Training College, University of Bamenda, P.O. Box 39, Bamenda, Cameroon \\ ${ }^{3}$ Research Group on Experimental and Applied Physics for Sustainable Development, Faculty of Science, Department of Physics, \\ University of Dschang, P.O. Box 412, Dschang, Cameroon \\ ${ }^{4}$ Laboratory of Electronics and Signal Processing Faculty of Science, Department of Physics, University of Dschang, \\ P.O. Box 67, Dschang, Cameroon \\ ${ }^{5}$ Laboratory of Modelling and Simulation in Engineering, Biomimetics and Prototypes (LaMSEBP) and TWAS Research Unit, \\ Department of Physics, Faculty of Science, University of Yaoundé I, P.O. Box 812, Yaoundé, Cameroon
}

Correspondence should be addressed to Gaetan Fautso Kuiate; fautso_kuiate@yahoo.com

Received 16 June 2017; Accepted 9 August 2017; Published 12 October 2017

Academic Editor: Karthikeyan Rajagopal

Copyright (c) 2017 Sifeu Takougang Kingni et al. This is an open access article distributed under the Creative Commons Attribution License, which permits unrestricted use, distribution, and reproduction in any medium, provided the original work is properly cited.

\begin{abstract}
A linear resistive-capacitive-inductance shunted junction (LRCLSJ) model obtained by replacing the nonlinear piecewise resistance of a nonlinear resistive-capacitive-inductance shunted junction (NRCLSJ) model by a linear resistance is analyzed in this paper. The LRCLSJ model has two or no equilibrium points depending on the dc bias current. For a suitable choice of the parameters, the LRCLSJ model without equilibrium point can exhibit regular and fast spiking, intrinsic and periodic bursting, and periodic and chaotic behaviors. We show that the LRCLSJ model displays similar dynamical behaviors as the NRCLSJ model. Moreover the coexistence between periodic and chaotic attractors is found in the LRCLSJ model for specific parameters. The lowest order of the commensurate form of the no equilibrium LRCLSJ model to exhibit chaotic behavior is found to be 2.934. Moreover, adaptive finite-time synchronization with parameter estimation is applied to achieve synchronization of unidirectional coupled identical fractional-order form of chaotic no equilibrium LRCLSJ models. Finally, a cryptographic encryption scheme with the help of the finite-time synchronization of fractional-order chaotic no equilibrium LRCLSJ models is illustrated through a numerical example, showing that a high level security device can be produced using this system.
\end{abstract}

\section{Introduction}

A Josephson junction (JJ) is made up of two superconductors, separated by a nonsuperconducting layer so thin that electrons can cross through the insulating barrier [1]. The flow of current between the superconductors in the absence of an applied voltage is called a Josephson current and the movement of electrons across the barrier is known as Josephson tunneling $[2,3]$. The JJ finds practical use as a millimetre or submillimetre wave oscillator [4], in digital systems [5] and in superconducting quantum interference devices such as magnetometer applications [6]. Based on the rapid development of superconducting devices in the fabrication technology different models of JJ have been reviewed in order to understand whether such superconducting junction can be used for ultrahigh-speed chaotic generators for applications of code generation in spread-spectrum communications and random key generation in secure communication and encryption [7]. 


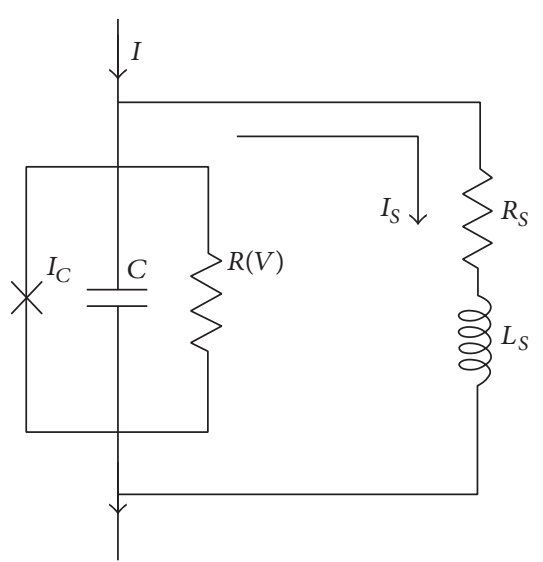

(a)

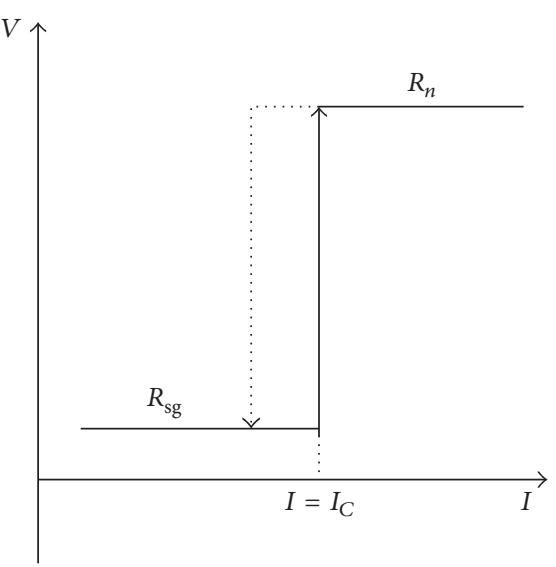

(b)

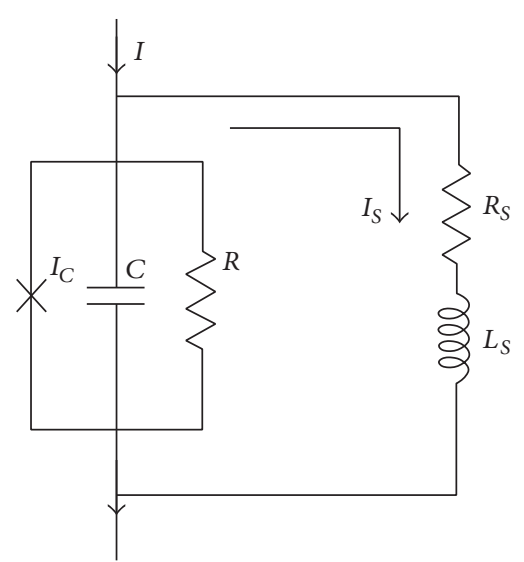

(c)

Figure 1: (a) The schematic representation of NRCLSJ model [14]. (b) Current-voltage characteristics at a temperature T (in Kelvin) of the intrinsic junction shunt resistance $R(V)$ [3]. (c) The schematic representation of LRCLSJ model.

In the literature, we can find different models of JJ, namely, linear resistive-capacitive shunted junction (RCSJ) model, nonlinear resistive-capacitive shunted junction model, and the NRCLSJ model [3, 8-12]. The first two models show chaotic behaviors when driven by an external sinusoidal signal [11] while the NRCLSJ model generates chaotic oscillation with external dc bias only [12-14]. Regular spiking, intrinsic bursting, and fast spiking which are usually seen in the mammalian neocortex have been found in the NRCLSJ model [15]. For large inductance, the NRCLSJ model behaves as a relaxation oscillator [16]. The NRCLSJ model $[8,9]$ is used to simulate JJ, resulting in a fairly good agreement with experiment. The RCSJ model, however, fails to reproduce significant features on experimental $I-V$ curves when the shunt of the JJ contains an inductance component $[12,17]$.

In this paper, we use the NRCLSJ model where the nonlinear piecewise resistance is replaced by a linear resistance to make it the LRCLSJ model. In [18], Neumann and Pikovsky reported on the study of a nontrivial type of slow-fast dynamics in LRCLSJ model. However, to the best of our knowledge, the dynamical behavior of the LRCLSJ model without equilibrium and its fractional-order form remains unaddressed. It is important to note that, due to the absence of equilibrium, the LRCLSJ model belongs to a class of systems with hidden attractors [19-22]. Systems with hidden attractors have three different families: systems with an infinite number of equilibrium points, systems with only stable equilibrium point, and systems without equilibrium points. A hidden attractor has a basin of attraction that does not intersect with small neighborhoods of any equilibrium points, while a self-excited attractor has a basin of attraction that is associated with an unstable equilibrium. Almost all famous chaotic attractors are self-excited. Hidden attractors are of high interest in engineering applications because they can exhibit unexpected and potentially disastrous responses to perturbations in a structure like a bridge or an airplane wing [23-26]. The paper is articulated around four sections presented as follows: in Section 2, the analytical and numerical analysis of the LRCLSJ model are investigated. In
Section 3, we focus on the dynamical behavior, synchronization, and application to digital cryptography in the fractionalorder form of LRCLSJ model without equilibrium points. Finally the conclusion of the paper is drawn in Section 4.

\section{Rate-Equations and Analysis of the Linear Resistive-Capacitive-Inductance Shunted Junction Model}

In this work, we consider the NRCLSJ model where the nonlinear resistance $R(V)$ is replaced by a linear resistance $R$ as shown in Figure 1.

The NRCLSJ model is presented in Figure 1(a). The intrinsic junction shunt nonlinear resistance $R(V)$ is modelled by a piecewise linear resistor as shown in Figure 1(b). In Figure 1(c), the shunted nonlinear resistance in Figure 1(a) is replaced by a linear resistor $R$. The JJ is represented by the supercurrent channel $I_{C} . I$ is the bias current applied to the JJ and $C$ is the junction capacitance. A current $I_{S}$ flows through the shunt inductance $L_{S}$ and its internal resistance $R_{S}$. The application of the Kirchhoff laws to the circuit of Figure 1(c) leads to the following differential equation:

$$
\begin{aligned}
I & =C \frac{d V}{d t^{\prime}}+\frac{V}{R}+I_{C} \sin \phi+I_{S} \\
V & =L_{S} \frac{d I_{S}}{d t^{\prime}}+R_{S} I_{S} \\
V & =\frac{\hbar}{2 e} \frac{d \phi}{d t^{\prime}},
\end{aligned}
$$

where $\phi$ denotes the phase difference of JJ. Using the dimensionless variables,

$$
\begin{aligned}
& x=\frac{V}{R_{S} I_{C}}, \\
& y=\frac{I_{S}}{I_{C}}, \\
& z=\phi,
\end{aligned}
$$




$$
\begin{aligned}
t^{\prime} & =\frac{t}{\omega_{0}}, \\
\omega_{0} & =\frac{2 \pi e R_{S} I_{C}}{h}, \\
\beta_{R} & =\frac{R_{S}}{R}, \\
\beta_{C} & =\frac{2 \pi e C I_{C} R_{S}^{2}}{h}, \\
\beta_{L} & =\frac{2 \pi e L_{S} I_{C}}{h}, \\
i & =\frac{I}{I_{C}} .
\end{aligned}
$$

The dimensionless set of (1a)-(1c) can be rewritten as

$$
\begin{aligned}
& \frac{d x}{d t}=\frac{1}{\beta_{C}}\left(i-y-\beta_{R} x-\sin z\right) \\
& \frac{d y}{d t}=\frac{1}{\beta_{L}}(x-y) \\
& \frac{d z}{d t}=x,
\end{aligned}
$$

where $\beta_{R}, \beta_{C}, \beta_{L}, i$ are positive parameters which represent the $\mathrm{dc}$ bias current, the ratio of resistors, the capacitor, and the inductance, respectively. Therefore, the LRCLSJ model is described by the set of (3a)-(3c).

2.1. Analytical Analysis of the Linear Resistive-CapacitiveInductance Shunted Junction Model. System (3a)-(3c) is dissipative because $\nabla V=\partial \dot{x} / \partial x+\partial \dot{y} / \partial y+\partial \dot{z} / \partial z=-\left(\beta_{R} / \beta_{C}+\right.$ $\left.1 / \beta_{L}\right)<0$. For $i>1$, it does not have an equilibrium point, while, for $i \leq 1$, that same system has two equilibrium points $E_{1}=(0,0, \arcsin (i))$ and $E_{2}=(0,0, \pi-\arcsin (i))$. The characteristic equation associated with the equilibrium point $E=\left(x^{*}, y^{*}, z^{*}\right)$ is

$$
\begin{aligned}
\lambda^{3}+ & \left(\frac{\beta_{R}}{\beta_{C}}+\frac{1}{\beta_{L}}\right) \lambda^{2} \\
& +\left[\frac{1}{\beta_{C} \beta_{L}}+\frac{\beta_{R}}{\beta_{C} \beta_{L}}+\frac{1}{\beta_{C}} \cos \left(z^{*}\right)\right] \lambda \\
& +\frac{1}{\beta_{C} \beta_{L}} \cos \left(z^{*}\right)=0,
\end{aligned}
$$

which, for $E_{1}$, gives

$$
\begin{aligned}
\lambda^{3}+ & \left(\frac{\beta_{R}}{\beta_{C}}+\frac{1}{\beta_{L}}\right) \lambda^{2} \\
& +\left[\frac{1}{\beta_{C} \beta_{L}}+\frac{\beta_{R}}{\beta_{C} \beta_{L}}+\frac{1}{\beta_{C}} \cos (\arcsin (i))\right] \lambda \\
& +\frac{1}{\beta_{C} \beta_{L}} \cos (\arcsin (i))=0
\end{aligned}
$$

and, for $E_{2}$, the characteristic equation becomes

$$
\begin{aligned}
\lambda^{3}+ & \left(\frac{\beta_{R}}{\beta_{C}}+\frac{1}{\beta_{L}}\right) \lambda^{2} \\
& +\left[\frac{1}{\beta_{C} \beta_{L}}+\frac{\beta_{R}}{\beta_{C} \beta_{L}}-\frac{1}{\beta_{C}} \cos (\arcsin (i))\right] \lambda \\
& -\frac{1}{\beta_{C} \beta_{L}} \cos (\arcsin (i))=0 .
\end{aligned}
$$

According to the Routh-Hurwitz criteria, the equilibrium point $E_{1}$ is a stable node and the equilibrium point $E_{2}$ is a saddle node.

2.2. Dynamical Behaviors of the Linear Resistive-CapacitiveInductance Shunted Junction Model. Dynamics of LRCLSJ model is investigated by considering the effect of parameters on system's behavior in order to see if it can exhibit some of the dynamical behaviors of the NRCLSJ model. Our simulations show that for $i<1.0$ the trajectories of system (3a)-(3c) converge to one of the equilibrium points $E_{1,2}$ while, for $i>1.0$, the trajectories of system (3a)-(3c) display periodic or complex behaviors as will be seen in the paragraphs below. It is interesting to note that, for $i>1.0$, system (3a)-(3c) has no equilibrium point. We find regular spiking, intrinsic bursting, fast spiking, and periodic bursting in the junction as shown in Figure 2 when the capacitive parameter $\beta_{C}$ is kept fixed at $\beta_{C}=0.007$, while the dc bias $i$, the inductive $\beta_{L}$, and resistive $\beta_{R}$ parameters are varied.

For $\beta_{L}=1.5, \beta_{R}=0.1$, and $i=1.25$, the junction exhibits regular spiking as shown in Figure 2(a). The regular spiking is sensitive to dc bias $i$ and the parameter $\beta_{R}$. In Figure 2(b), for the same values of $\beta_{L}$ and $\beta_{R}$ parameters, intrinsic bursting is observed for larger dc bias current $i=$ 1.75 when the spiking frequency is obviously larger. For $\beta_{L}=$ 0.5 , the fast spiking is found in Figure 2(c) when the dc bias current $i$ and other parameters remain the same as for intrinsic bursting. For $\beta_{R}=0.01$, the periodic bursting is observed in Figure 2(d) when the dc bias current $i$ and other parameters are kept fixed as for regular spiking. Such spiking behaviors have been found in the NRCLSJ model [16] and are typical of the mammalian neocortex [27]. As in the NRCLSJ model, the LRCLSJ model behaves as a relaxation oscillator for large value of parameter $\beta_{L}$ [15]. This is shown in Figure 3 which presents the time series of $x(t)$ and corresponding phase portrait for $\beta_{L}=26, \beta_{C}=0.5, i=1.25$, and $\beta_{R}=0.1$.

In the following, the dynamical behavior of system (3a)-(3c) is illustrated using bifurcation diagrams, Lyapunov exponent, time series, phase portraits, and basin of attraction. In Figure 4, we plot the bifurcation diagram depicting the local maxima of $x(t)$ as a function of dc bias current $i$ for $\beta_{L}=2.6, \beta_{C}=0.707$, and $\beta_{R}=0.06$.

When the dc bias current $i$ increases from 1.05 to 2.1, the bifurcation diagram of Figure 4(a) shows period-2oscillations followed by an intermittency route to chaos interspersed with periodic windows. For $i>1.255$, a reverse period-tripling bifurcation to period-2-oscillations at $i \approx 1.594$ is observed. By increasing the dc bias current $i$, the output $x(t)$ displays period-2-oscillations followed by 

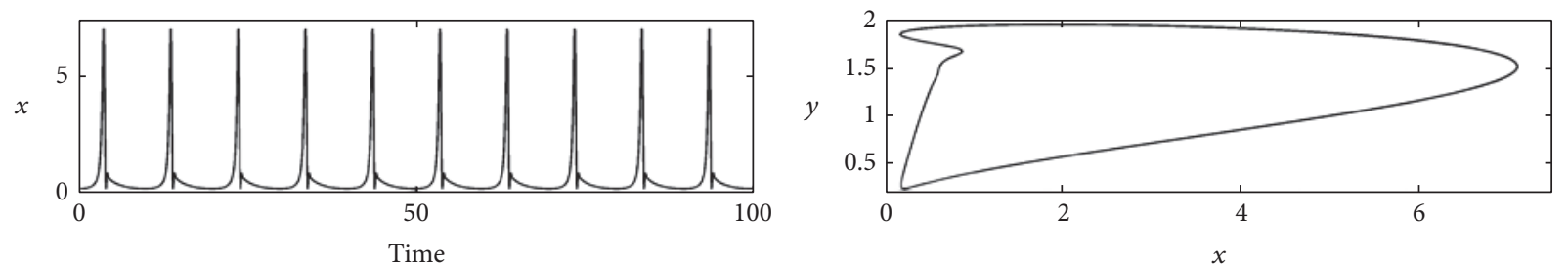

(a)
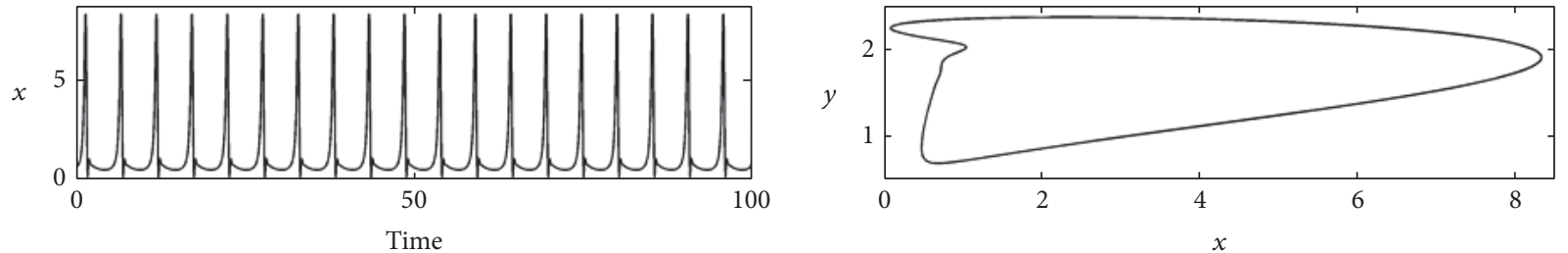

(b)
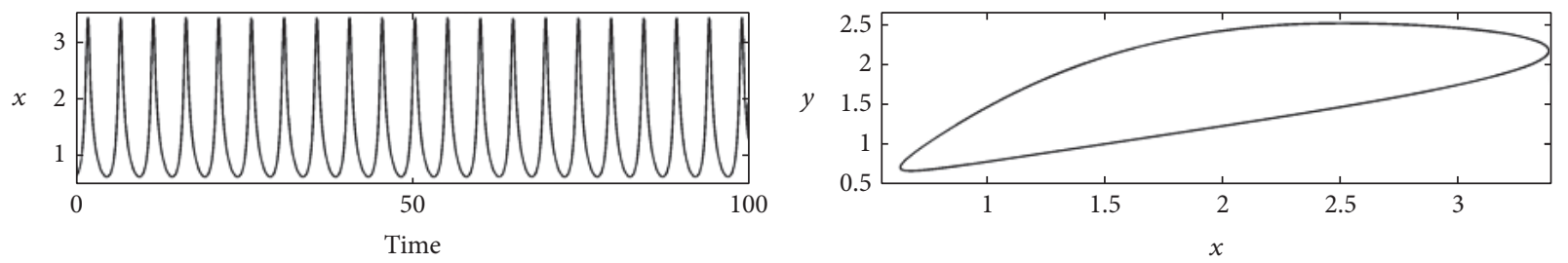

(c)
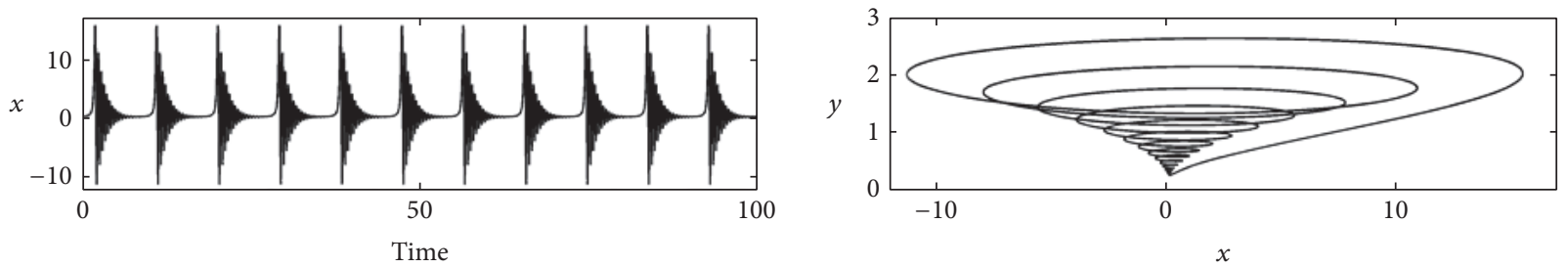

(d)

FIGURE 2: Time series of $x(t)$ and corresponding phase portraits of system (3a) $-(3 \mathrm{c})$ in plane $(x, y)$ for specific values of (a) $\beta_{L}=1.5, i=1.25$, and $\beta_{R}=0.1$, (b) $\beta_{L}=1.5, i=1.75$, and $\beta_{R}=0.1$, (c) $\beta_{L}=0.5, i=1.75$, and $\beta_{R}=0.1$, and (d) $\beta_{L}=1.5, i=1.25$, and $\beta_{R}=0.01$. The other parameter is $\beta_{C}=0.007$.

period-1-oscillations for $i \geq 2.0085$. The chaotic behavior is confirmed by the largest Lyapunov exponent shown in Figure 4(b). The chaotic behavior is illustrated in Figure 5 for a specific value of dc bias current $i$.

From Figure 5, we observe that system (3a)-(3c) exhibits chaotic attractor similar to the one found in the NRCLSJ model [12-16].

For $i=1.25, \beta_{C}=0.707$, and $\beta_{R}=0.06$, we plot the bifurcation diagram depicting the local maxima of $x(t)$ and the largest Lyapunov exponent of system (3a)-(3c) versus the parameter $\beta_{L}$ as shown in Figure 6.

When the parameter $\beta_{L}$ increases from 1.9 to 2.8 [see black dot in Figure 6(a)], the bifurcation diagram of the output $x(t)$ shows period-2-oscillations followed by an intermittency route to chaos interspersed with periodic windows. For $\beta_{L}>2.625$, a reverse period-tripling bifurcation to period-3oscillations at $\beta_{L} \approx 2.725$ is observed. Period-3-oscillations persists for $\beta_{L}>2.725$. When performing the same analysis by ramping the parameter $\beta_{L}$ [see red dot in Figure 6(a)], the output $x(t)$ displays the same dynamical behaviors as in
Figure 6(a) (see black dot) in the ranges $1.9 \leq \beta_{L}<2.014$ and $2.0725<\beta_{L} \leq 2.8$, while, in the range $2.014 \leq \beta_{L} \leq$ 2.0725 , the output $x(t)$ shows period-1-oscillations, perioddoubling bifurcation, and chaotic oscillations, respectively. By comparing the two sets of data [for increasing (black) and decreasing (red)] used to plot Figure 6(a), one can notice that system (3a)-(3c) displays coexistence of attractors in the range $2.014 \leq \beta_{L} \leq 2.0725$. For $2.014 \leq \beta_{L}<2.0375$, period2-oscillations coexist with period-1-oscillations. Period-2oscillations coexist with period-doubling bifurcation in the range $2.0375 \leq \beta_{L}<2.056$. For $2.056<\beta_{L} \leq 2.0725$, period1-oscillations coexist with chaotic oscillations. The chaotic behavior is confirmed by the largest Lyapunov exponent shown in Figure 6(b). The coexistence of attractors found in Figure 6(a) is illustrated in Figure 7 which depicts the phase portraits and cross section of the basin of attraction of system (3a) $-(3 c)$ for specific value of parameter $\beta_{L}$.

System (3a)-(3c) exhibits period-1-oscillations and chaotic attractor for $\beta_{L}=2.06$ and depending on the initial conditions as shown in Figure 7(a). One can see from 

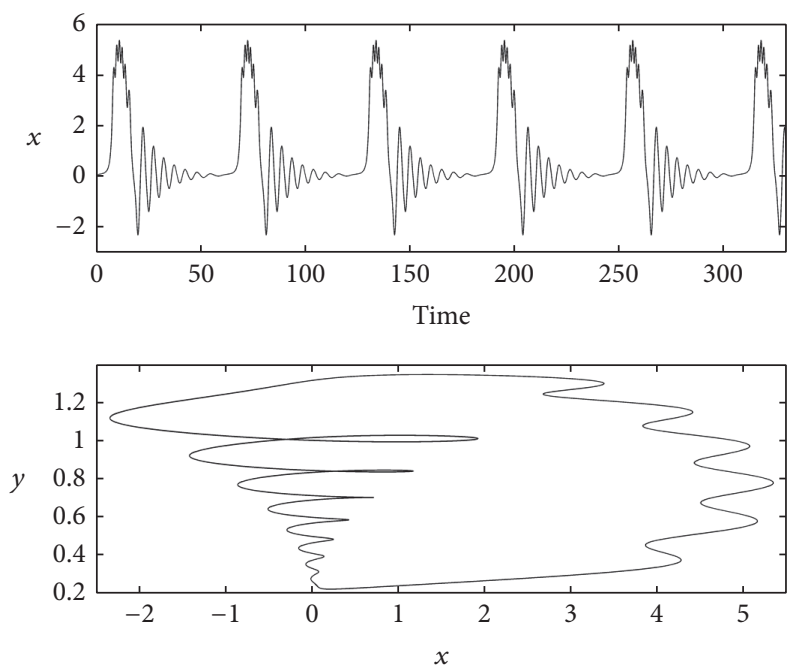

FIGURE 3: Time series of $x(t)$ and corresponding phase portrait of system (3a)-(3c) in plane $(x, y)$ for $\beta_{L}=26, \beta_{C}=0.5, i=1.25$, and $\beta_{R}=0.1$.

Figure $7(a)$ that system (3a)-(3c) can exhibit either chaotic or periodic attractor depending on the initial conditions. It is worth noting that coexistence of attractors has been observed in various nonlinear systems including laser [28, 29], biological system [30], chemical reactions [31], Lorenz systems [32-35], and electrical circuits [36, 37], just to name a few.

\section{Fractional-Order Form of Chaotic No Equilibrium Linear Resistive-Capacitive- Inductance Shunted Junction Model}

In this section, we consider the commensurate fractionalorder form of the no equilibrium LRCLSJ model given by

$$
\begin{aligned}
\frac{d^{q} x}{d t^{q}} & =\frac{1}{\beta_{C}}\left(i-y-\beta_{R} x-\sin z\right) \\
\frac{d^{q} y}{d t^{q}} & =\frac{1}{\beta_{L}}(x-y) \\
\frac{d^{q} z}{d t^{q}} & =x,
\end{aligned}
$$

where $q$ is the derivative order satisfying $0<q \leq$ 1. For numerical solutions of the above set of commensurate fractional-order differential equations, the Adams Bashforth-Moulton predictor-corrector scheme [38] is used. This method is based on the Caputo definition of the fractional-order derivative, given by $[39,40]$

$$
\frac{d^{q} X_{i}}{d t^{q}}=\frac{1}{\Gamma(q-n)} \int_{0}^{t} \frac{X_{i}^{(n)}\left(t^{\prime}\right)}{\left(t-t^{\prime}\right)^{q-n+1}} d t^{\prime},
$$

where $n-1<q<n, X_{1}=x, X_{2}=y, X_{3}=z$, and $\Gamma(\cdot)$ is the Gamma function. In the coming subsections, we

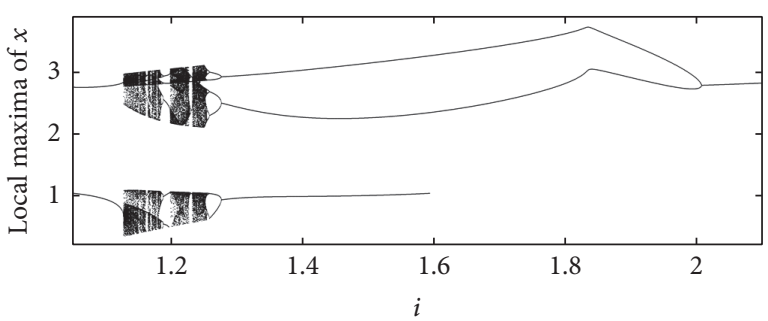

(a)

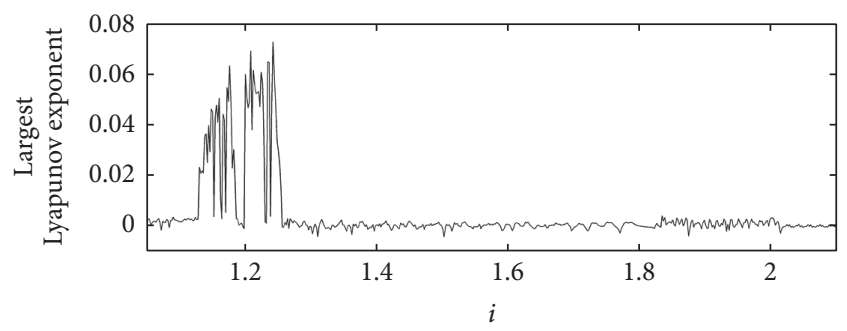

(b)

FIGURE 4: The bifurcation diagram depicting the local maxima of $x(t)$ (a) and the largest Lyapunov exponent (b) of system (3a)-(3c) versus the dc bias current $i$ for $\beta_{L}=2.6, \beta_{C}=0.707$, and $\beta_{R}=0.06$.

will focus on the effect of fractional derivation on the chaotic system (3a)-(3c) when $i=1.25, \beta_{L}=2.6, \beta_{C}=0.707$, and $\beta_{R}=0.06$ (see Figure 5). Chaos synchronization of unidirectional identical coupled commensurate fractionalorder system (7a)-(7c) and engineering applications shall also be investigated.

3.1. Effect of Commensurate Fractional Derivation on the Chaotic No Equilibrium Linear Resistive-CapacitiveInductance Shunted Junction Model. For the dc bias current $i>1$, the commensurate fractional-order system (7a)-(7c) has no equilibrium points. Therefore we cannot determine analytically the lowest order of the commensurate fractionalorder system (7a)-(7c) to exhibit chaotic behavior. Rather, the effect of commensurate fractional derivation on chaotic system (3a)-(3c) for $i=1.25, \beta_{L}=2.6, \beta_{C}=0.707$, and $\beta_{R}=0.06$ can only be numerically investigated. Here accordingly, using the Adams Bashforth-Moulton predictorcorrector scheme presented in this subsection, we plot the bifurcation diagram showing the local maxima of the state variable $x$ with respect to the commensurate fractionalorder $q$ as shown in Figure 8 in order to find the lowest order of system (7a)-(7c) to remain chaotic.

The bifurcation diagram of Figure 8 indicates period2-oscillations followed by chaotic behavior for $q \geq 0.978$. Hence, the lowest order for the commensurate fractionalorder form of the no equilibrium LRCLSJ model to show chaos is $3 q \approx 2.934$. In Figure 9 , the time series of $x(t)$ and the phase portraits in the plane $(x, y)$ of significant results was obtained for specific values of commensurate fractionalorder $q$.

For $q=0.98$, Figure 9 (a) shows chaotic attractor. Period-2-oscillations (see Figure 9(b)), regular spiking (see 

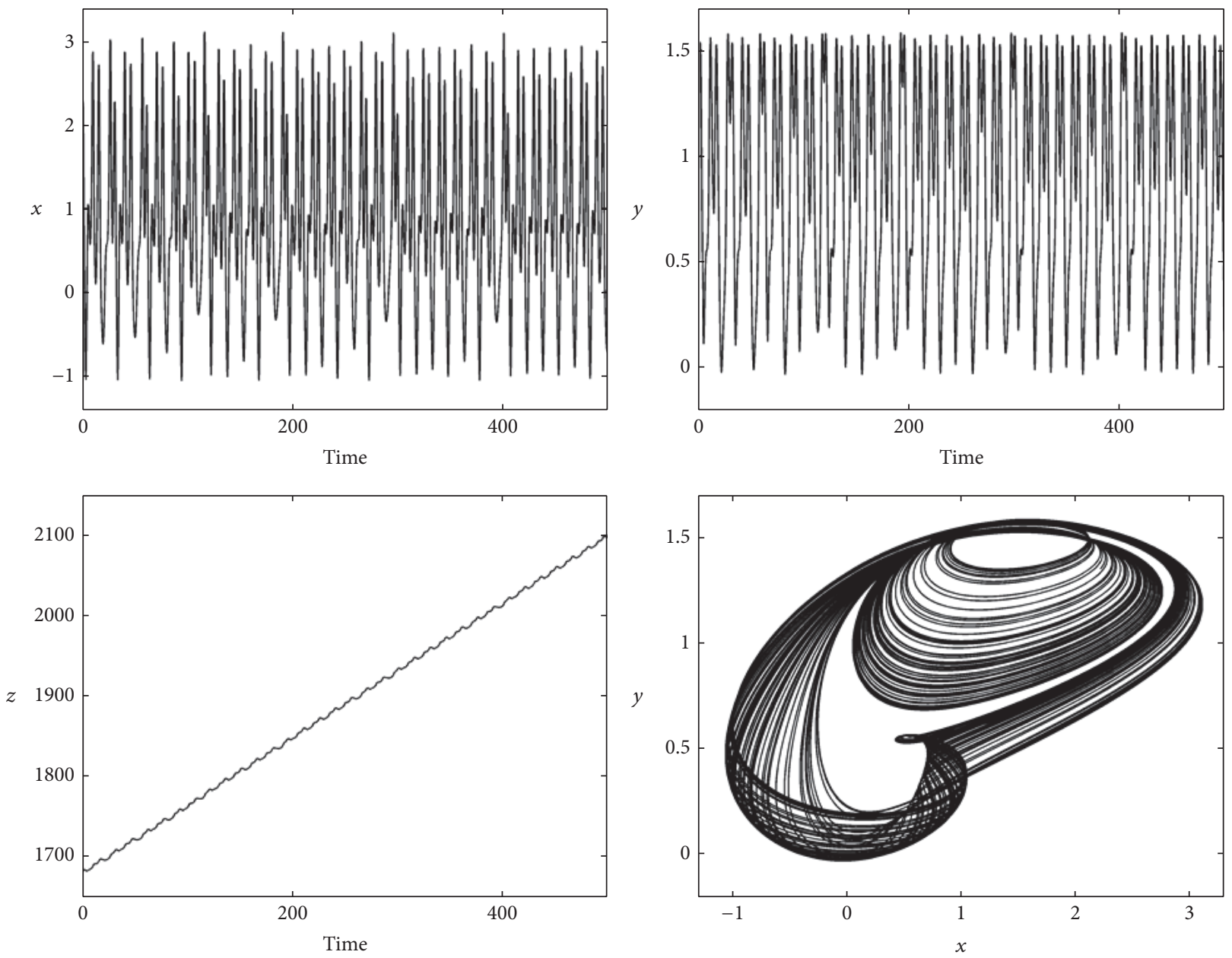

FIGURE 5: Time series of $x(t), y(t)$, and $z(t)$ and corresponding phase portrait of system (3a) $-(3 \mathrm{c})$ in plane $(x, y)$ for $i=1.25$. The other parameters are $\beta_{L}=2.6, \beta_{C}=0.707$, and $\beta_{R}=0.06$. The initial conditions are $(x(0), y(0), z(0))=(0.0,0.0,1.0)$.

Figure $9(\mathrm{c})$ ), and fast spiking (see Figure $9(\mathrm{~d})$ ) are shown at $q=0.96, q=0.8$, and $q=0.7$, respectively.

\subsection{Adaptive Finite-Time Synchronization with Parameter} Estimation of Unidirectional Coupled Identical FractionalOrder Form Chaotic No Equilibrium Linear ResistiveCapacitive-Inductance Shunted Junction Models. In many practical cases, it is difficult (or impossible) to accurately determine the values of the parameters of dynamical systems to be synchronized. As it was proven that accurate control of these parameters can significantly affect the synchronization process, however, this problem can be solved by adaptive synchronization with parameter estimation [41]. The aim of this subsection is to provide an example of adaptive finitetime synchronization with parameter estimation applied to the chaotic commensurate fractional-order system (7a)-(7c). The drive system can be written in the form

$$
\begin{aligned}
& \frac{d^{q} x_{1}}{d t^{q}}=\frac{1}{\beta_{C}}\left(i-y_{1}-\beta_{R} x_{1}-\sin z_{1}\right) \\
& \frac{d^{q} y_{1}}{d t^{q}}=\frac{1}{\beta_{L}}\left(x_{1}-y_{1}\right)
\end{aligned}
$$

$$
\frac{d^{q} z_{1}}{d t^{q}}=x_{1}
$$

And the response system is described by

$$
\begin{aligned}
\frac{d^{q} x_{2}}{d t^{q}} & =\frac{1}{\beta_{C}}\left(i-y_{2}-\widehat{\beta}_{R} x_{2}-\sin z_{2}-s(t)\right) \\
\frac{d^{q} y_{2}}{d t^{q}} & =\frac{1}{\beta_{L}}\left(x_{2}-y_{2}\right) \\
\frac{d^{q} z_{2}}{d t^{q}} & =x_{2}
\end{aligned}
$$

The controller signal is $s(t)=\left(z_{2}-z_{1}\right)+k u(t)$, where $u(t)$ is the adaptive feedback coupling designed to achieve finite-time synchronization. We set the synchronization error between drive and response systems as $e_{x}=x_{2}-x_{1}, e_{y}=$ $y_{2}-y_{1}, e_{z}=z_{2}-z_{1}$, and $e_{\beta}=\widehat{\beta}_{R}-\beta_{R}$. The errors system is obtained as follows: 


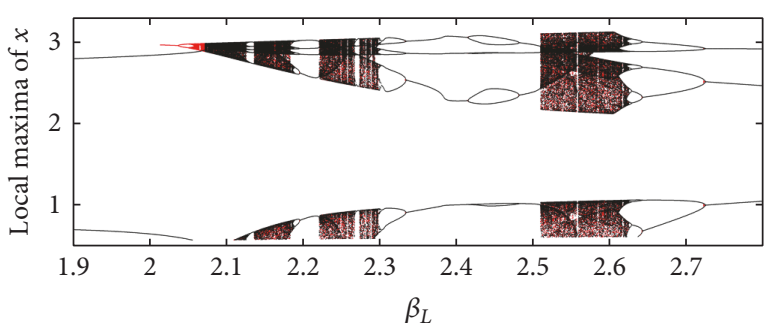

(a)

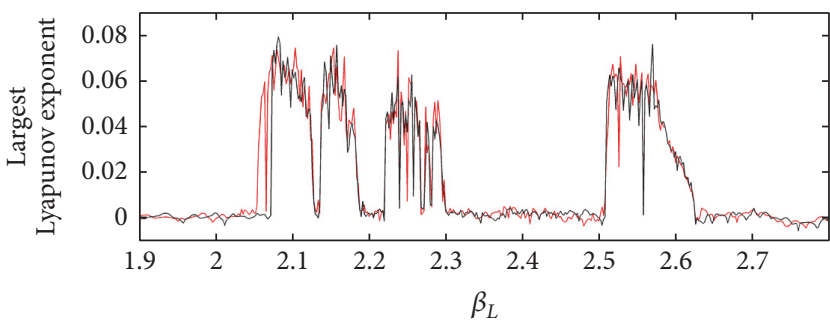

(b)

Figure 6: The bifurcation diagrams depicting the local maxima of $x(t)$ (a) and the largest Lyapunov exponent (b) of system (3a)-(3c) versus the parameter $\beta_{L}$ for $i=1.25, \beta_{C}=0.707$, and $\beta_{R}=0.06$. Bifurcation diagrams are obtained by scanning the parameter $\beta_{L}$ upwards (black) and downwards (red).

$$
\begin{aligned}
& \frac{d^{q} e_{x}}{d t^{q}} \\
& =\frac{1}{\beta_{C}}\left(-e_{y}-x_{2} e_{\beta}-\beta_{R} e_{x}-f\left(z_{1}, z_{2}\right)-e_{z}-k u(t)\right) \\
& \frac{d^{q} e_{y}}{d t^{q}}=\frac{1}{\beta_{L}}\left(e_{x}-e_{y}\right) \\
& \frac{d^{q} e_{z}}{d t^{q}}=e_{y}
\end{aligned}
$$

with the nonlinear function $f\left(z_{1}, z_{2}\right)$ defined by $f\left(z_{1}, z_{2}\right)=$ $\sin z_{2}-\sin z_{1}$. The response system (10a)-(10c) synchronizes with the drive system $(8)$ at a finite-time and final time $t_{\text {syn }}$ exists such that $\lim _{t \rightarrow t_{\text {syn }}}\left\|e_{i}(t)\right\|=0$.

Assumption 1. The nonlinear function $f\left(z_{1}, z_{2}\right)$ with $f(0$, $0)=0$ respects the Lipschitz condition, if and only if scalar numbers $\eta_{1}$ and $\eta_{2}$ exist such that $\left\|f\left(z_{1}, z_{2}\right)\right\| \leq \eta_{1}\left\|e_{z}\right\| \leq$ $\eta_{2}\left\|e_{x}\right\|$.

Theorem 2. The response system (10a)-(10c) synchronizes with the drive system (8) in the finite-time

$$
\begin{aligned}
t_{\text {syn }}^{q} \leq & t_{\text {syn }}^{1} \\
= & \frac{1}{2 \chi}\left(\beta_{C} e_{x}^{2}(0)+\beta_{L} e_{y}^{2}(0)+e_{z}^{2}(0)+e_{\beta}^{2}(0)\right) \\
& +\frac{|u(0)|}{\chi} .
\end{aligned}
$$

If the adaptive feedback controller and the estimated parameter are set as follows:

$$
\begin{aligned}
& \frac{d^{q} \widehat{\beta}_{R}}{d t^{q}}=x_{2} e_{x} \\
& \frac{d^{q} u}{d t^{q}}=\left(k u e_{x}-\eta e_{x}^{2}-\chi\right) \operatorname{sign}(u),
\end{aligned}
$$

where $\chi, \eta$, and $k$ are the positive parameters. The slave system can be also written as

$$
\begin{aligned}
& \frac{d^{q} x_{2}}{d t^{q}} \\
& \quad=\frac{1}{\beta_{C}}\left(i-y_{2}-\widehat{\beta}_{R} x_{2}-\sin z_{2}-\left(z_{2}-z_{1}\right)-k u\right) \\
& \frac{d^{q} y_{2}}{d t^{q}}=\frac{1}{\beta_{L}}\left(x_{2}-y_{2}\right) \\
& \frac{d^{q} z_{2}}{d t^{q}}=x_{2} \\
& \frac{d^{q} \widehat{\beta}_{R}}{d t^{q}}=x_{2} e_{x} \\
& \frac{d^{q} u}{d t^{q}}=\left(k u e_{x}-\eta e_{x}^{2}-\chi\right) \operatorname{sign}(u) .
\end{aligned}
$$

Proof. We consider the following Lyapunov candidate function defined by

$$
V(t)=\frac{1}{2}\left(\beta_{C} e_{x}^{2}+\beta_{L} e_{y}^{2}+e_{z}^{2}+e_{\beta}^{2}\right)+|u(t)| .
$$

The fractional-order derivative of (15) gives

$$
\begin{aligned}
\frac{d^{q} V(t)}{d t^{q}}= & -x_{2} e_{x} e_{\beta}-\beta_{R} e_{x}^{2}-f\left(z_{1}, z_{2}\right) e_{x}-k u e_{x}-e_{y}^{2} \\
& +e_{\beta} \frac{d^{q} \widehat{\beta}_{R}}{d t^{q}}+\operatorname{sign}(u) \frac{d^{q} u}{d t^{q}} .
\end{aligned}
$$

Using the assumption, (16) becomes $d^{q} V(t) / d t^{q} \leq-x_{2} e_{x} e_{\beta}+$ $\eta e_{x}^{2}-k u e_{x}+e_{\beta} D^{q} \widehat{\beta}_{R}+\operatorname{sign}(u)\left(d^{q} u / d t^{q}\right)$. If a positive constant $\chi$ exists such that $d^{q} V(t) / d t^{q} \leq-\chi$, then the update laws for the controller $u(t)$ and the estimated parameter $\widehat{\beta}_{R}$ are designed through the following relations:

$$
\begin{aligned}
& \frac{d^{q} \widehat{\beta}_{R}}{d t^{q}}=x_{2} e_{x} \\
& \frac{d^{q} u}{d t^{q}}=\left(k u e_{x}-\eta e_{x}^{2}-\chi\right) \operatorname{sign}(u) .
\end{aligned}
$$

In [42], the authors have shown that the finite-time synchronization of fractional-order system is shorter than the finite-time synchronization of integer-order systems. This implies that the finite-time of synchronization for integerorder systems is also finite-time of synchronization for the fractional-order version of the same systems with the same 


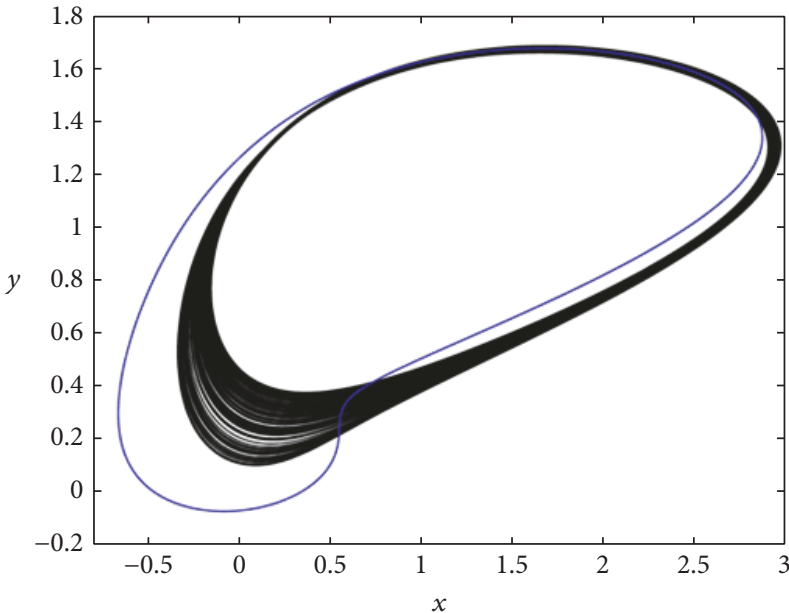

(a)

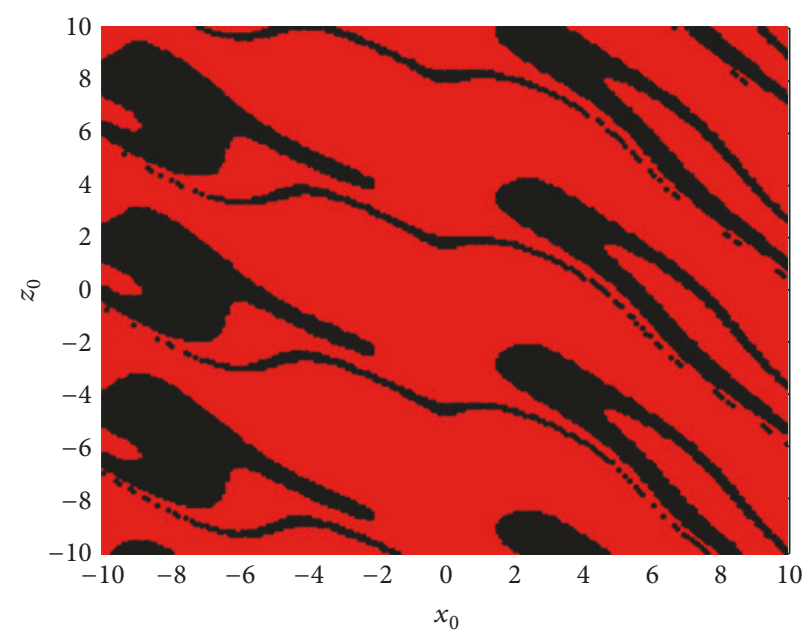

(b)

Figure 7: (a) Phase portraits in the plane $(x, y)$ for specific initial conditions: (blue line) $(x(0), y(0), z(0))=(0.0,0.0,1.0)$ and (black line) $(x(0), y(0), z(0))=(3.3,0.0,1.0)$. (b) Cross section of the basin of attraction of system (3a)-(3c) in the $x z$-plane at $y=0$. In subplot (b), periodic attractors are in red color and chaotic attractors are in black color. The parameters values are $\beta_{L}=2.06, i=1.25, \beta_{C}=0.707$, and $\beta_{R}=0.06$.

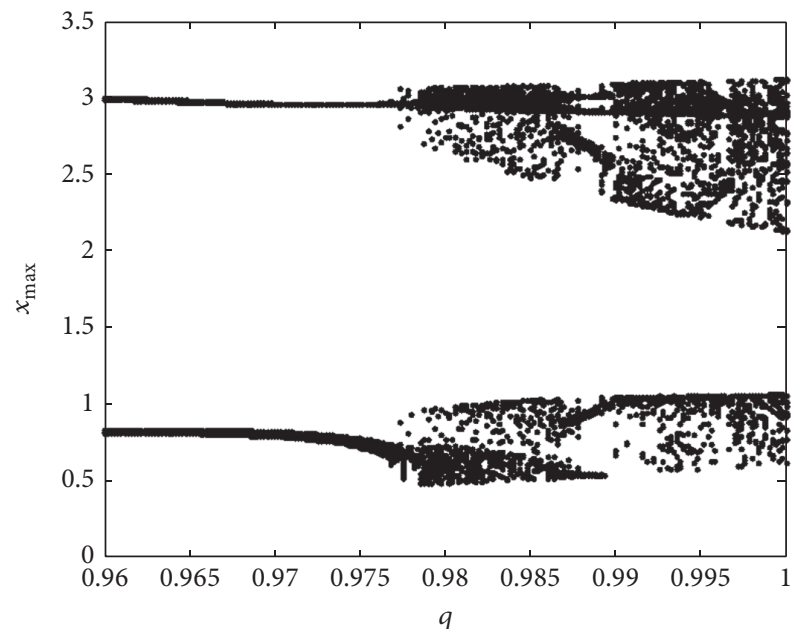

FIGURE 8: Bifurcation diagram showing the local maxima of the state variable $x$ with respect to the commensurate fractional-order $q$. The parameters are set as $i=1.25, \beta_{L}=2.6, \beta_{C}=0.707$, and $\beta_{R}=0.06$.

controller involved. Therefore the fractional-order response system (10a)-(10c) synchronizes with the drive system (8) in a finite-time of synchronization given by (12). Figures 10 and 11 are the graphical representation of the synchronization process obtained numerically by carrying out a numerical integration of drive system described by (8) and the response system (10a)-(10c) for their respective initial conditions chosen as $\left(x_{1}(0), y_{1}(0), z_{1}(0)\right)=(0,0,1)$ and $\left(x_{2}(0), y_{2}(0), z_{2}(0), \widehat{\beta}_{R}(0), u(0)\right)=(0.1,0.1,1.2,-0.3,0.1)$. To guarantee the chaotic synchronization, the other parameters are fixed at $q=0.98, \beta_{C}=00.707, i=1.25, \beta_{L}=2.6$, $\beta_{R}=0.06, \eta=0.01, \chi=0.004$, and $k=3$. Based on these values, the theoretical settled time of synchronization is $t_{\text {Tsyn }}^{1}=45.375$.
The dynamic of the synchronization error is present in Figure 11.

From Figure 11, it can be seen that the numerical time of synchronization is $t_{N \text { syn }}^{q} \approx 40$. By comparing with analytical time of synchronization obtained above, it can be noted that $t_{N \text { syn }}^{q} \leq t_{T \text { syn }}^{1}$. This result respects the finite-time condition given in [42]. Thus, these results can be used for chaos based communications.

3.3. Application to Digital Cryptography. In this subsection, we propose a digital cryptography scheme based on the adaptive finite-time synchronization with parameters estimation of unidirectional coupled identical commensurate fractional-order form chaotic no equilibrium LRCLSJ models developed in the previous subsection. The technique of digital cryptography to be used in the present paper is an improved version of the method developed and implemented in [4244] but we improve these methods. In [42], the authors used the adaptive finite-time synchronization with parameter estimation for message encryption; meanwhile in the present work we use the adaptive finite-time synchronization with parameters estimation which is recognized to be more robust than its counterpart [41]. Cryptography is a way to encode a message by changing its original message form into another different message whose conversion rule is known only by the sender. In the digital cryptography, for instance, the letters can be replaced by numbers which can be assigned by the sender. In this work, the message (plaintext) will be replaced by their ASCII codes before the encryption. As the ASCII code table has 128 characters, we define the formula for the ciphertext and decrypted message corresponding to the assignment of numbers as follows:

$$
\begin{aligned}
c & =p_{s}+k_{s} \quad(\bmod 128) \\
p_{r} & =c-k_{r} \quad(\bmod 128),
\end{aligned}
$$



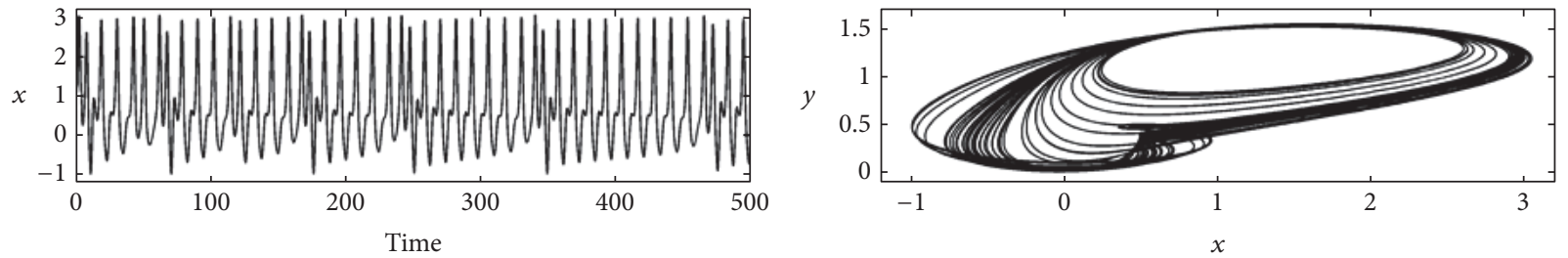

(a)
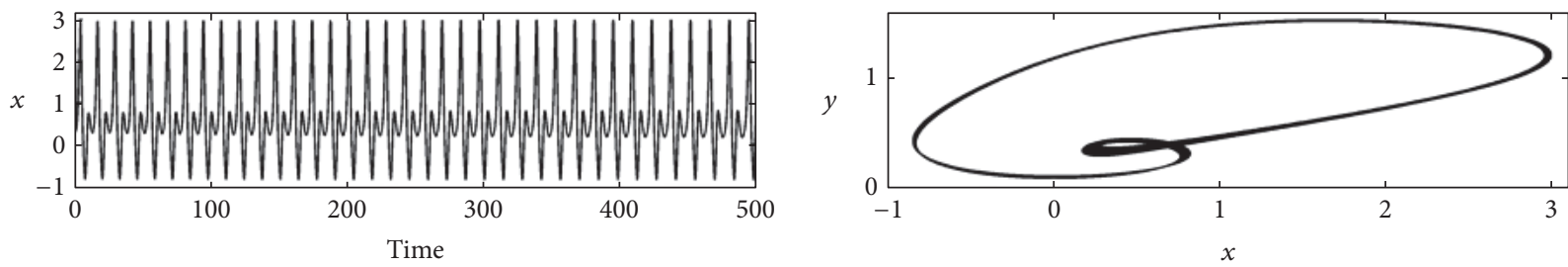

(b)
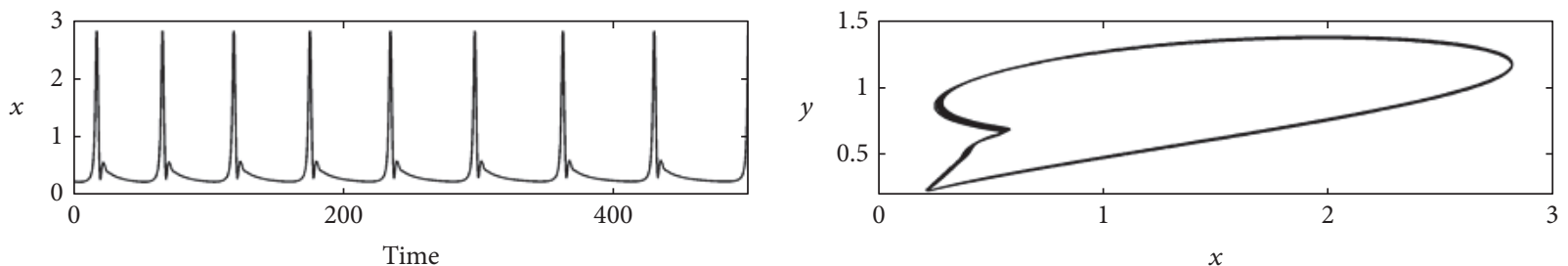

(c)
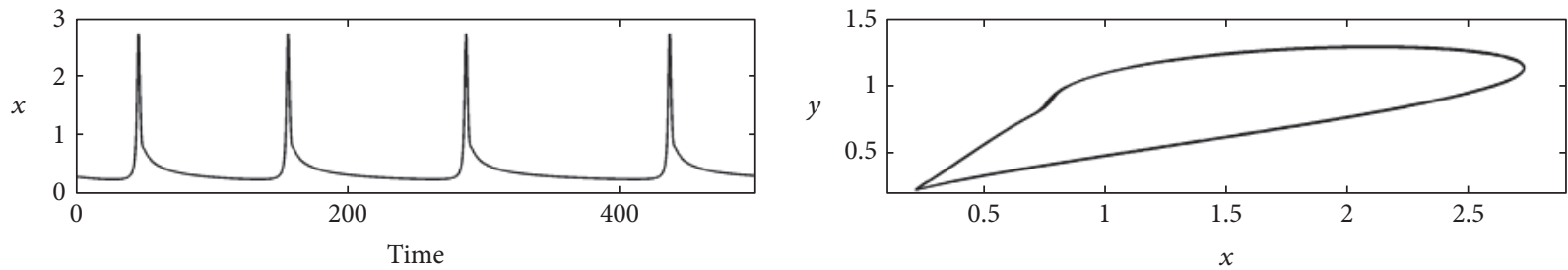

(d)

FIGURE 9: Time series of $x(t)$ and corresponding phase portraits in plane $(x, y)$ of the fractional-order system $(7 \mathrm{a})-(7 \mathrm{c})$ with commensurate fractional-orders: (a) $q=0.98$, (b) $q=0.96$, (c) $q=0.8$, and (d) $q=0.7$. The other parameters are $\beta_{L}=2.6, \beta_{C}=0.707, i=1.25$, and $\beta_{R}=0.06$. The initial conditions are $(x(0), y(0), z(0))=(0,0,1)$.

where $p_{s}$ is the plaintext to be encrypted, $p_{r}$ is the plaintext to be recovered, $k_{s}$ is the secret keys of sender, $k_{r}$ is the secret keys of the receiver, and $c$ is the ciphertext.

3.3.1. Proposed Affine Cipher. Consider $S$ and $R$, respectively, as the sender and the receiver in the cryptosystem. Also consider the drive system (8) as a sender's system and the response system (10a)-(10c) as a receiver's system. $S$ and $R$ agree on a time $t \geq t_{\text {syn }}^{q}$, fractional-order $q=0.98$, and a number of characters of the ASCII codes table equal to 128. The public key $D=$ Day/Month/year is only shared by $S$ and $R$. This public key allows building the two privates keys. The complete procedure between $S$ and $R$ is described as follows.

Key Generation. (1) The plaintext can be presented into a form of integers using the ASCII code table. After this assignation that plaintext can be organized in blocks of four terms. If the number of letters constituting the message is a multiple of 4 , we will have $p_{1}, p_{2}, p_{3}, p_{4}$ as a first bloc and $p_{5}, p_{6}, p_{7}, p_{8}$ as a second block and so on. On the other hand, if the number of letters of that message is not a multiple of 4 then blank spaces have to be insert at the end of the sequence to complete it to a multiple of 4 .

(2) For $t \geq t_{\text {syn }}^{q}$ and $q \geq 0.98$, the sender $S$ has $\left(x_{1}, y_{1}, z_{1}\right)$ as solution of commensurate fractional-order chaotic system (8), and the receive $R$ has $\left(x_{2}, y_{2}, z_{2}\right)$ as solution of system (10a)-(10c). The private keys of the sender and receiver are, respectively, defined as follows:

$$
\begin{array}{r}
k_{1}=\left[\text { Day } * z_{1}+\text { Month } * y_{1}+\text { year } * x_{1}\right] \\
(\bmod 128) \\
k_{2}=\left[\text { Day } * z_{2}+\text { Month } * y_{2}+\text { year } * x_{2}\right] \\
(\bmod 128),
\end{array}
$$

where $[a]$ is the integer part of $a$. 


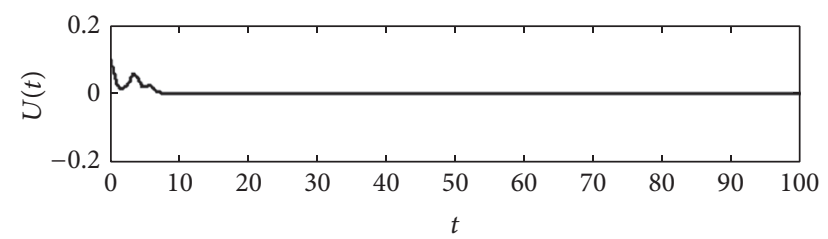

(a)

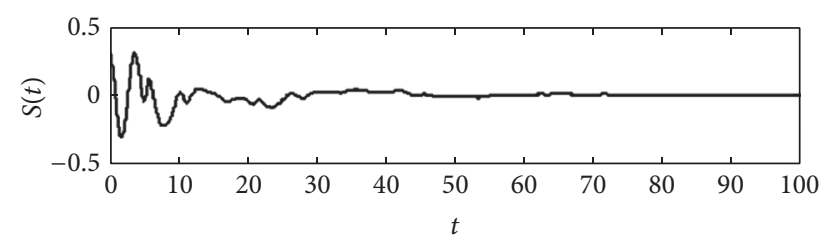

(b)

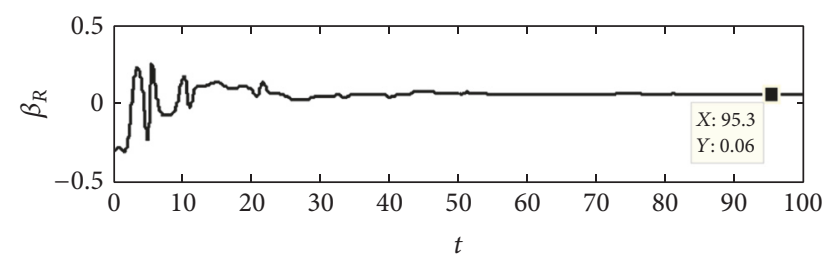

(c)

FIGURE 10: The time evolution of the adaptive feedback controller $u(t)$ (a) and the controller signal $S(t)(\mathrm{b})$ as well as the estimated parameter $\widehat{\beta}_{R}(\mathrm{c})$.
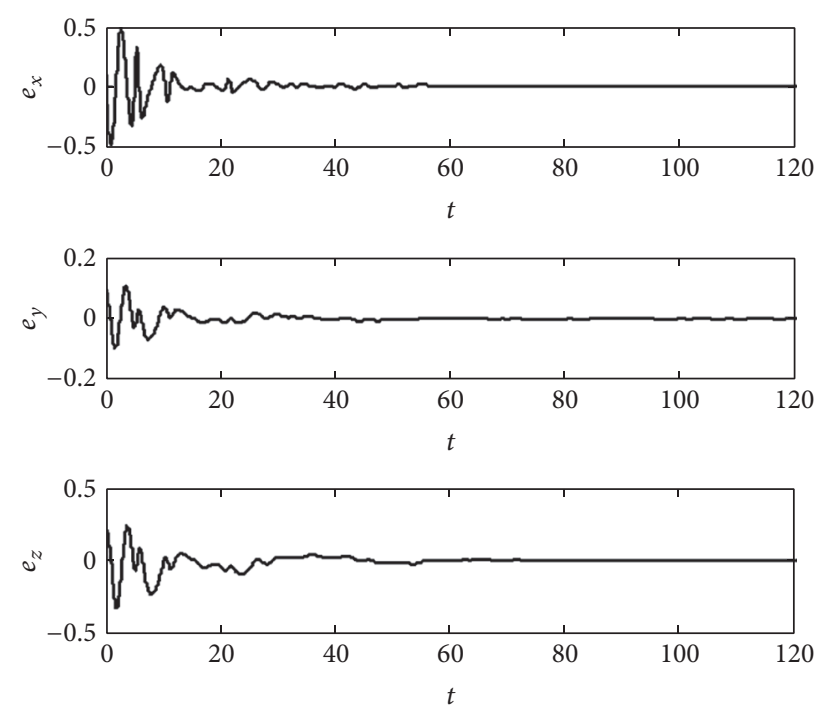

FIGURE 11: Synchronization errors between the drive and response systems (8) and (10a)-(10c). The parameters are given in the text.

(3) The first four sending keys $k_{1 s}, k_{2 s}, k_{3 s}, k_{4 s}$ are determined by

$$
Q^{k_{1}}=\left(\begin{array}{cc}
F_{k_{1}+1} & F_{k_{1}} \\
F_{k_{1}} & F_{k_{1}-1}
\end{array}\right)=\left(\begin{array}{ll}
\tilde{k}_{1} & \tilde{k}_{2} \\
\tilde{k}_{3} & \widetilde{k}_{4}
\end{array}\right),
$$

where $k_{1 s}=\widetilde{k}_{1}(\bmod 128), k_{2 s}=\widetilde{k}_{2}(\bmod 128), k_{3 s}=\widetilde{k}_{3}$ $(\bmod 128), k_{4 s}=\widetilde{k}_{4}(\bmod 128)$, and $F_{n}$ is the Fibonacci number; $F_{n+1}=F_{n}+F_{n-1}$ with $n \geq 3$ taking into account the fact that the initial terms are $F_{0}=0$ and $F_{1}=F_{2}=0$.

(4) The first four receiving keys $k_{1 r}, k_{2 r}, k_{3 r}, k_{4 r}$ are determined by

$$
Q^{k_{2}}=\left(\begin{array}{cc}
F_{k_{2}+1} & F_{k_{2}} \\
F_{k_{2}} & F_{k_{2}-1}
\end{array}\right)=\left(\begin{array}{ll}
\tilde{k}_{1} & \tilde{k}_{2} \\
\tilde{k}_{3} & \widetilde{k}_{4}
\end{array}\right),
$$

where $k_{1 r}=\widetilde{k}_{1}(\bmod 128), k_{2 r}=\widetilde{k}_{2}(\bmod 128), k_{3 r}=\widetilde{k}_{3}$ $(\bmod 128)$, and $k_{4 r}=\widetilde{k}_{4}(\bmod 128)$.

(5) The ciphertext and recovered plaintext are obtained by applying $c=p_{s}+k_{s}(\bmod 128)$ and $p_{r}=c-k_{r}(\bmod 128)$, respectively. After the treatment of the first block of plaintext we restart the process in the next block and so on.

As an example, we want to send the message PASSWORD to a third party. By following the five steps mentioned above, we obtain the summarized results in Table 1.

3.3.2. Security Analysis of Proposed Affine Cipher. The present proposed affine cipher consists of three keys: one public key $D$ and two private keys $k_{1}$ and $k_{2}$. The two private keys have been computed with the public key. The four receive keys and four sending keys have been computed with the private and sending keys as well as the Fibonacci numbers. The receiver cannot recover an original text without the knowledge of the receiver private key $k_{2}$. Compared to the existing work in this field to the best of our knowledge, our proposed technique is fully authenticated and more adapted for applications in telecommunications because we have used the adaptive finite-time synchronization with parameters estimation. In fact more recently in [42], the authors used the adaptive finite-time synchronization to improve the digital cryptography developed in $[43,44]$. Yet, the adaptive finitetime synchronization is less robust than the adaptive finitetime synchronization with parameters estimation. The finitetime adaptive synchronization based on the identification of system parameters appears to be of great practical interest, especially when the system state is available to external measurements [45]. Therefore, the proposed affine cipher is more efficient than the ordinary affine cipher method due to the adaptive finite-time synchronization with parameters estimation.

\section{Conclusion}

The present study dealt with the analysis of the linear resistive-capacitive-inductance shunted junction model and its fractional-order form. The linear resistive-capacitiveinductance junction shunted model has two or no equilibrium points depending on the dc bias current. By using the Routh-Hurwitz stability criteria, it has been found that the two equilibrium points are stable and saddle nodes, respectively. In spite of the absence of equilibrium, the linear resistive-capacitive-inductance shunted junction model can exhibit fast and regular spiking, intrinsic and periodic bursting, periodic and chaotic behaviors, and coexistence of attractors. Then, the commensurate fractional-order form of chaotic no equilibrium linear resistive-capacitiveinductance shunted junction model has been investigated. 
TABLE 1: Summary of sending and receive message (PASSWORD).

\begin{tabular}{|c|c|c|c|c|c|c|c|}
\hline Times & $k_{1}$ & Plaintext & Keys $k_{s}$ & Ciphertext & $k_{2}$ & Keys $k_{r}$ & Plaintext recovered \\
\hline \multirow{4}{*}{$t=80$} & \multirow{4}{*}{55} & $80(\mathrm{P})$ & 23 & 103 & \multirow{4}{*}{55} & 23 & $80(\mathrm{P})$ \\
\hline & & $65(\mathrm{~A})$ & 34 & 99 & & 34 & $65(\mathrm{~A})$ \\
\hline & & $83(S)$ & 34 & 117 & & 34 & $83(S)$ \\
\hline & & $83(\mathrm{~S})$ & 117 & 200 & & 117 & $83(\mathrm{~S})$ \\
\hline \multirow{4}{*}{$t=100$} & \multirow{4}{*}{56} & $87(\mathrm{~W})$ & 57 & 144 & \multirow{4}{*}{56} & 57 & $87(W)$ \\
\hline & & $79(\mathrm{O})$ & 23 & 102 & & 23 & $79(\mathrm{O})$ \\
\hline & & $82(\mathrm{R})$ & 23 & 105 & & 23 & $82(\mathrm{R})$ \\
\hline & & $68(\mathrm{D})$ & 34 & 102 & & 34 & $68(\mathrm{D})$ \\
\hline
\end{tabular}

Chaos has been shown to exist in commensurate fractionalorder form of no equilibrium linear resistive-capacitiveinductance shunted junction model with orders less than 3. Using adaptive finite-time synchronization with parameter estimation, chaos synchronization has been found between unidirectional coupled identical fractional-order forms of chaotic no equilibrium linear resistive-capacitive-inductance shunted junction models. Applying the synchronized commensurate fractional-order form of chaotic no equilibrium linear resistive-capacitive-inductance shunted junction models in digital cryptography, a well secured key system has been obtained.

\section{Conflicts of Interest}

The authors declare that there are no conflicts of interest regarding the publication of this paper.

\section{Acknowledgments}

Sifeu Takougang Kingni would like to thank Dr. Victor Kamdoum Tamba (University of Dschang, Cameroon) and Dr. Bonaventure Nana (University of Bamenda, Cameroon) for stimulating discussions.

\section{References}

[1] B. Soodchomshom, I.-M. Tang, and R. Hoonsawat, "Josephson effects in $\mathrm{MgB}_{2}$ /Thin Insulator/ $\mathrm{MgB}_{2}$ tunnel junction," Solid State Communications, vol. 149, no. 25-26, pp. 1012-1016, 2009.

[2] B. D. Josephson, "Possible new effects in superconductive tunnelling," Physics Letters, vol. 1, no. 7, pp. 251-253, 1962.

[3] J. Clarke, "A superconducting galvanometer employing Josephson tunnelling," Philosophical Magazine, vol. 13, no. 121, pp. 115127, 1966.

[4] V. K. Kornev and A. V. Arzumanov, "Josephson-junction oscillation spectral linewidth for some phase-locked multijunction systems," Le Journal de Physique IV, vol. 8, no. 3, pp. Pr3-279Pr3-282, 1998.

[5] A. Kanasugi, M. Morisue, H. Noguchi, M. Yamadaya, and H. Furukawa, "Oscillation modes in a josephson circuit and its application to digital systems," IEICE Transactions on Electronics, vol. E79-C, no. 9, pp. 1206-1211, 1996.

[6] J. W. Spargo, "Applied superconductivity conference," IEEE Transactions on Applied Superconductivity, vol. 13, pp. I-III, 2003.
[7] R. Guo, U. E. Vincent, and B. A. Idowu, "Synchronization of chaos in RCL-shunted Josephson junction using a simple adaptive controller," Physica Scripta, vol. 79, no. 3, Article ID 035801, 2009.

[8] W. C. Stewart, "Current-voltage characteristics of Josephson junctions," Applied Physics Letters, vol. 12, no. 8, pp. 277-280, 1968.

[9] D. E. McCumber, "Effect of ac impedance on dc voltage-current characteristics of superconductor weak-link junctions," Journal of Applied Physics, vol. 39, no. 7, pp. 3113-3118, 1968.

[10] M. Levi, F. C. Hoppensteadt, and W. L. Miranker, "Dynamics of the Josephson junction," Quarterly of Applied Mathematics, vol. 36, no. 2, pp. 167-198, 1978/79.

[11] F. M. Salam and S. S. Sastry, "Dynamics of the forced Josephson junction circuit: the regions of chaos," Institute of Electrical and Electronics Engineers. Transactions on Circuits and Systems, vol. 32, no. 8, pp. 784-796, 1985.

[12] C. B. Whan, C. J. Lobb, and M. G. Forrester, "Effect of inductance in externally shunted Josephson tunnel junctions," Journal of Applied Physics, vol. 77, no. 1, pp. 382-389, 1995.

[13] C. B. Whan and C. J. Lobb, "Complex dynamical behavior in ", Physical Review E, vol. 53, no. 1, pp. 405-413, 1996.

[14] A. B. Cawthorne, C. B. Whan, and C. J. Lobb, "Complex dynamics of resistively and inductively shunted Josephson junctions," Journal of Applied Physics, vol. 84, no. 2, pp. 11261132, 1998.

[15] S. K. Dana, D. C. Sengupta, and K. D. Edoh, "Chaotic dynamics in Josephson Junction," IEEE Transactions on Circuits and Systems I: Fundamental Theory and Applications, vol. 48, no. 8, pp. 990-996, 2001.

[16] S. K. Dana, "Spiking and Bursting in Josephson Junction," IEEE Transactions on Circuits and Systems II: Express Briefs, vol. 53, no. 10, pp. 1031-1034, 2006.

[17] A. D. Smith, R. D. Sandell, A. H. Silver, and J. F. Burch, "Chaos and Bifurcation in Josephson Voltage-Controlled Oscillators," IEEE Transactions on Magnetics, vol. 23, no. 2, pp. 1267-1270, 1987.

[18] E. Neumann and A. Pikovsky, "Slow-fast dynamics in Josephson junctions," European Physical Journal B, vol. 34, no. 3, pp. 293303, 2003.

[19] G. A. Leonov, N. V. Kuznetsov, and V. I. Vagaitsev, "Localization of hidden Chua's attractors," Physics Letters. A, vol. 375, no. 23, pp. 2230-2233, 2011.

[20] G. A. Leonov, N. V. Kuznetsov, and V. I. Vagaitsev, "Hidden attractor in smooth Chua systems," Physica D. Nonlinear Phenomena, vol. 241, no. 18, pp. 1482-1486, 2012. 
[21] G. A. Leonov and N. V. Kuznetsov, "Hidden attractors in dynamical systems. From hidden oscillations in HilbertKolmogorov, Aizerman, and KALman problems to hidden chaotic attractor in Chua circuits," International Journal of Bifurcation and Chaos in Applied Sciences and Engineering, vol. 23, no. 1, Article ID 1330002, 69 pages, 2013.

[22] G. A. Leonov, N. V. Kuznetsov, M. A. Kiseleva, E. P. Solovyeva, and A. M. Zaretskiy, "Hidden oscillations in mathematical model of drilling system actuated by induction motor with a wound rotor," Nonlinear Dynamics, vol. 77, no. 1-2, pp. 277-288, 2014.

[23] G. A. Leonov, N. V. Kuznetsov, and T. N. Mokaev, "Hidden attractor and homoclinic orbit in Lorenz-like system describing convective fluid motion in rotating cavity," Communications in Nonlinear Science and Numerical Simulation, vol. 28, no. 1-3, pp. 166-174, 2015.

[24] G. A. Leonov, N. V. Kuznetsov, and T. N. Mokaev, "Homoclinic orbits, and self-excited and hidden attractors in a Lorenz-like system describing convective fluid motion," European Physical Journal: Special Topics, vol. 224, no. 8, pp. 1421-1458, 2015.

[25] P. R. Sharma, M. D. Shrimali, A. Prasad, N. V. Kuznetsov, and G. A. Leonov, "Controlling dynamics of hidden attractors," International Journal of Bifurcation and Chaos in Applied Sciences and Engineering, vol. 25, no. 4, Article ID 1550061, 1550061, 7 pages, 2015.

[26] D. Dudkowski, S. Jafari, T. Kapitaniak, N. V. Kuznetsov, G. A. Leonov, and A. Prasad, "Hidden attractors in dynamical systems," Physics Reports. A Review Section of Physics Letters, vol. 637, pp. 1-50, 2016.

[27] E. M. Izhikevich, "Simple model of spiking neurons," IEEE Transactions on Neural Networks, vol. 14, no. 6, pp. 1569-1572, 2003.

[28] C. Masoller, "Coexistence of attractors in a laser diode with optical feedback from a large external cavity," Physical Review A, vol. 50, no. 3, pp. 2569-2578, 1994.

[29] S. T. Kingni, G. V. D. Sande, I. V. Ermakov, and J. Danckaert, "Theoretical analysis of semiconductor ring lasers with short and long time-delayed optoelectronic and incoherent feedback," Optics Communications, vol. 341, pp. 147-154, 2015.

[30] J. M. Cushing, S. M. Henson, and C. . Blackburn, "Multiple mixed-type attractors in a competition model," Journal of Biological Dynamics, vol. 1, no. 4, pp. 347-362, 2007.

[31] A. Massoudi, M. G. Mahjani, and M. Jafarian, "Multiple attractors in Koper-Gaspard model of electrochemical periodic and chaotic oscillations," Journal of Electroanalytical Chemistry, vol. 647, no. 1, pp. 74-86, 2010.

[32] C. Li, J. C. Sprott, and H. Xing, "Constructing chaotic systems with conditional symmetry," Nonlinear Dynamics, vol. 87, no. 2, pp. 1351-1358, 2017.

[33] C. Li, J. C. Sprott, and H. Xing, "Hypogenetic chaotic jerk flows," Physics Letters. A, vol. 380, no. 11-12, pp. 1172-1177, 2016.

[34] C. Li, W. Hu, J. C. Sprott, and X. Wang, "Multistability in symmetric chaotic systems," European Physical Journal: Special Topics, vol. 224, no. 8, pp. 1493-1506, 2015.

[35] C. Li, J. C. Sprott, and H. Xing, "Crisis in amplitude control hides in multistability," International Journal of Bifurcation and Chaos in Applied Sciences and Engineering, vol. 26, no. 14, Article ID 1650233, 1650233, 11 pages, 2016.

[36] J. Kengne, "Coexistence of chaos with hyperchaos, period-3 doubling bifurcation, and transient chaos in the hyperchaotic oscillator with gyrators," International Journal of Bifurcation and
Chaos in Applied Sciences and Engineering, vol. 25, no. 4, Article ID 1550052, 17 pages, 2015.

[37] A. P. Kuznetsov, S. P. Kuznetsov, E. Mosekilde, and N. V. Stankevich, "Co-existing hidden attractors in a radio-physical oscillator system," Journal of Physics A: Mathematical and Theoretical, vol. 48, no. 12, 12 pages, 2015.

[38] K. Diethelm, N. J. Ford, and A. D. Freed, "A predictor-corrector approach for the numerical solution of fractional differential equations," Nonlinear Dynamics. An International Journal of Nonlinear Dynamics and Chaos in Engineering Systems, vol. 29, no. 1-4, pp. 3-22, 2002.

[39] R. Caponetto, R. Dongola, L. Fortuna, I. Petra, and I. Petraš, "Fractional-order system: modelling and control applications. World scientific series on nonlinear science," series A, vol. 72, 2010.

[40] R. Hilfer, Applications of Fractional Calculus in Physics, World Scientific, Singapore, 2000.

[41] H. B. Fotsin and J. Daafouz, "Adaptive synchronization of uncertain chaotic colpitts oscillators based on parameter identification," Physics Letters, Section A: General, Atomic and Solid State Physics, vol. 339, no. 3-5, pp. 304-315, 2005.

[42] R. Kengne, R. Tchitnga, A. Mezatio, A. Fomethe, and G. Litak, "Finite-time synchronization of fractional-order simplest twocomponent chaotic oscillators," European Physical Journal B, vol. 90, pp. 88-96, 2017.

[43] P. Muthukumar and P. Balasubramaniam, "Feedback synchronization of the fractional order reverse butterfly-shaped chaotic system and its application to digital cryptography," Nonlinear Dynamics. An International Journal of Nonlinear Dynamics and Chaos in Engineering Systems, vol. 74, no. 4, pp. 1169-1181, 2013.

[44] P. Muthukumar, P. Balasubramaniam, and K. Ratnavelu, "Fast projective synchronization of fractional order chaotic and reverse chaotic systems with its application to an affine cipher using date of birth (DOB)," Nonlinear Dynamics, vol. 80, no. 4, pp. 1883-1897, 2015.

[45] W. W. Yu and J. D. Cao, "Adaptive synchronization and lag synchronization of uncertain dynamical system with time delay based on parameter identification," Physica A, vol. 375, no. 2, pp. 467-482, 2007. 


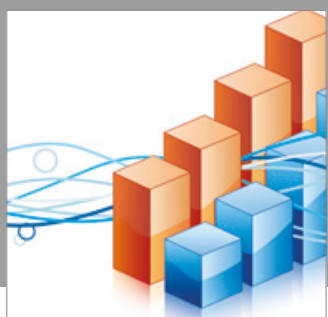

Advances in

Operations Research

vatersals

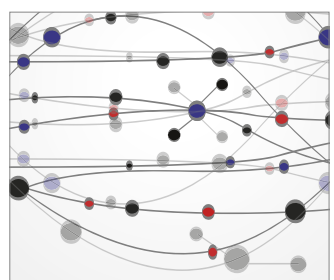

\section{The Scientific} World Journal
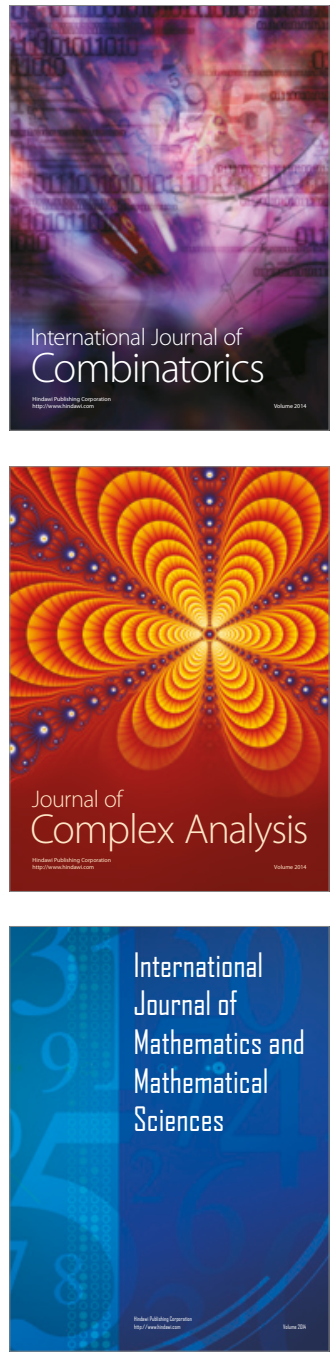
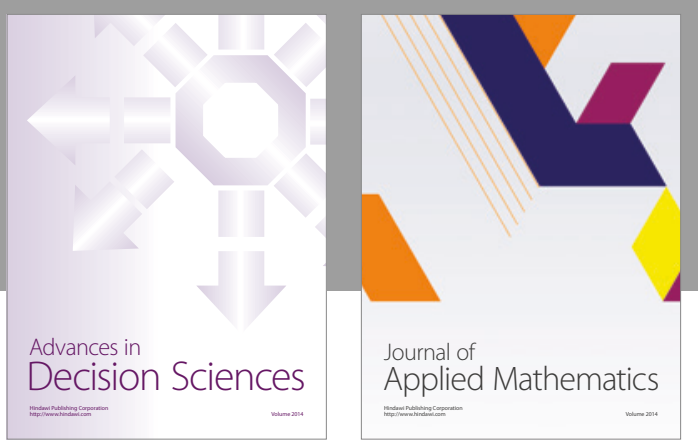

Algebra

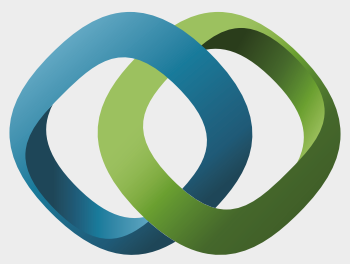

\section{Hindawi}

Submit your manuscripts at

https://www.hindawi.com
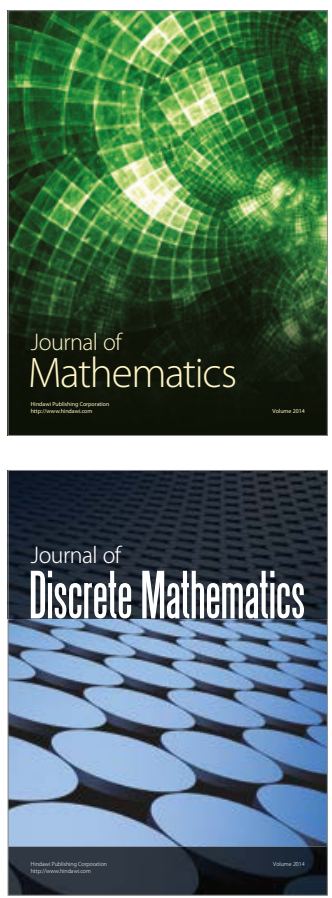

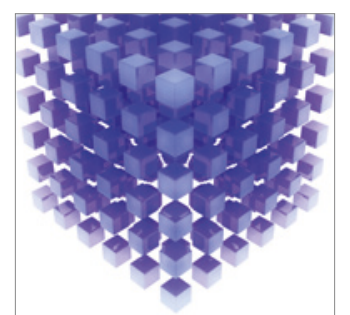

Mathematical Problems in Engineering
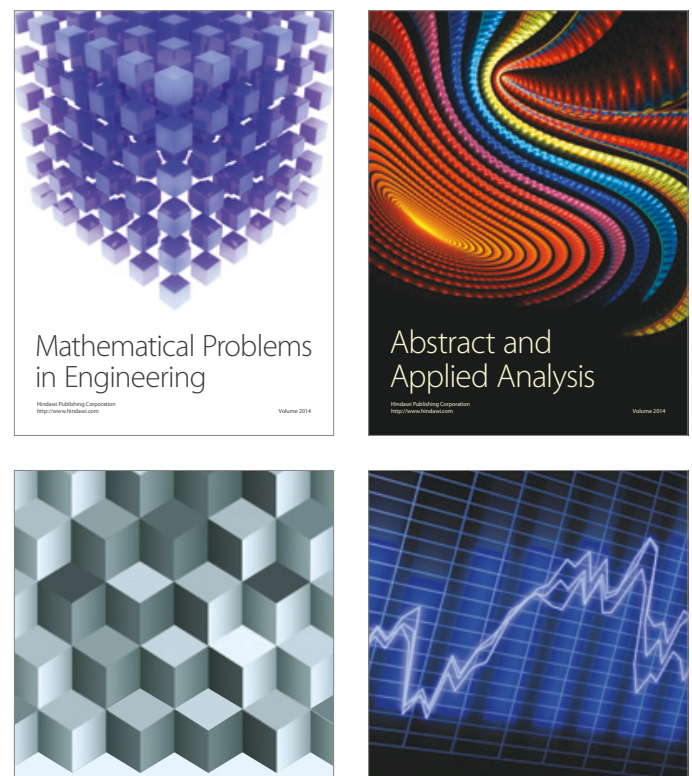

Journal of

Function Spaces

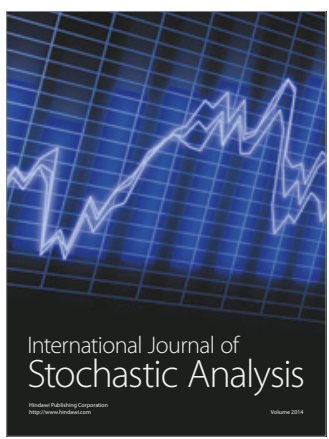

Probability and Statistics
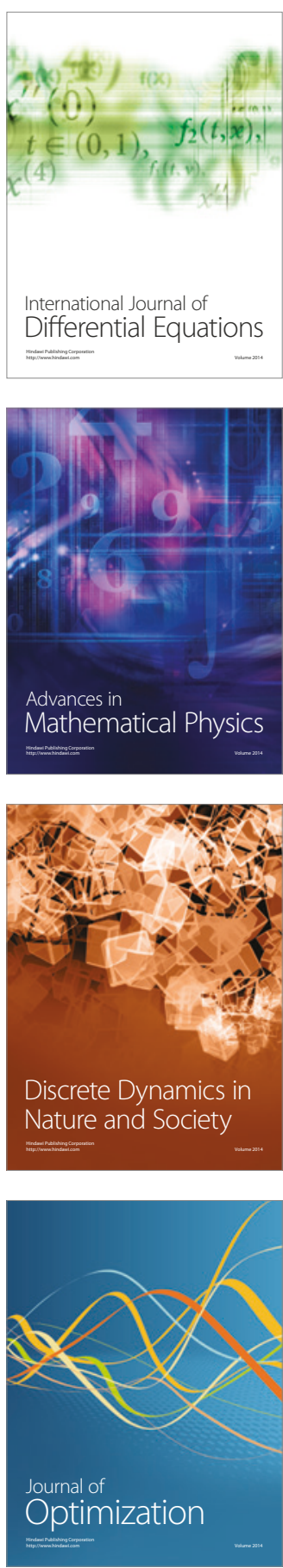